\title{
Research on Face Recognition Technology Based on Computer Neural Network
}

\author{
Yaning Yan \\ Xi'an Peihua University, Xi'an, 710125, China
}

Keywords: Face recognition, Computer neural network, Deep learning

\begin{abstract}
In recent years, face recognition technology has been used in more and more situations. In order to classify the feature vectors of the face image to achieve face recognition, a face recognition method using BP neural network is proposed in this paper. It described the basic process of face recognition algorithm based on BP neural network in detail, including face image detection, face image preprocessing, feature extraction of face image and matching and recognition of face image, to provide some references for the relevant researchers.
\end{abstract}

\section{Introduction}

Face recognition is a pattern recognition technology that uses computer to extract and recognize features of face images [1]. The research of face recognition began at the end of the 1960s. It is a hot topic in the field of Information Science in recent years. Face recognition technology has also been widely applied in social development and construction. In this paper, a face recognition algorithm based on graph matching is proposed, which extracts face recognition methods with node attribute, edge attribute and area attribute. The method needs to normalize the size of the sample before processing, and has a high recognition rate and strong anti-interference ability to the face image. Compared with the traditional face recognition method, it has the advantages of novel, simple and easy to realize, and the accuracy rate is very high. In recent years, the research of face recognition system has become very active in the field of computer vision and pattern recognition. As one of the important external identification features of human, face recognition is of great significance for automatic identification and human automatic resolution. Compared with fingerprint recognition and iris recognition, face recognition has its own particularity, that is, face image is influenced by external factors such as its imaging angle and illumination condition, so face recognition is more difficult. At present, the automatic face recognition system consists of two main technical links. The first is to detect and locate the face, that is to judge whether there is a face in an image. If there is, locate the specific position of each face. The two is the extraction and recognition of face features. It includes the location of the eyes, the nose, the mouth area and the extraction of the contour of the face image. Research shows that the feature extraction and recognition using BP neural network has been widely applied due to its good speed, recognition rate, fault tolerance and parallel computing power [2].

\section{Learning of BP Neural Networks}

\subsection{Concept of Artificial Neural Network}

Artificial neural network is the simplification, abstraction and Simulation of human brain, reflecting the basic characteristics of human brain. Neural network is a network which is widely interconnected by many processing units [3]. Neural network research is the function of simulating human brain information processing, from the physiological structure of human brain to study human intelligence behavior. The single layer forward network refers to the neuron with a single layer, and the input layer, as the number of the source nodes, is considered a layer of neurons. The input layer does not have the function of computing. The difference between multilayer feedforward neural network and single layer feedforward neural network is that the number of hidden layers of multilayer feedforward neural network and single layer forward neural network is different. The nodes that 
perform computing functions in multilayer feedforward neural networks are called hidden units. Because the number of hidden layers is different, the network can carry out higher order statistics. Especially when the input scale is large, the ability of hidden layer neurons to extract high order statistical data is very important. A feedback network refers to a neural network that contains at least one feedback loop in the network. The feedback network contains a single layer neuron. All neurons in this layer feed their output signals to all other neurons as inputs. Stochastic neural network introduces random concept in neural network, each neuron works according to the principle of probability, so that every neuron's excitation or inhibition is stochastic, and its probability depends on the input value of neurons. The most significant feature of the competitive neural network is that its output neurons compete to determine the winner [4].

\subsection{Learning of Neural Network}

BP neural network can learn and store many input - output model mapping relations, without the need to reveal the mathematical equation that describes the mapping relationship in advance. It uses adaptive learning algorithm to adjust the weights and thresholds of the network through reverse propagation, so that the sum of square errors of the network is minimized. The BP neural network model topology consists of the input layer, the hidden layer and the output layer. Neural network learning is also known as training. It refers to adjusting the parameters of neural network through the stimulation of the external environment of the neural network, so that the neural network can respond to the external environment in a well-regulated way. Learning from the environment and improving its performance in learning are the most significant features of the neural network. The way of learning can be divided into: learning by teachers and without teachers. Teacher learning, also known as supervised learning, needs to give guidance signals when learning. The neural network is unknown to the external environment, but the guiding signal can be regarded as an understanding of the external environment, which is represented by the set of input and output samples. The guidance signal or expected output represents the best result of the execution of neural network, that is, adjust the network parameters for network input, and make the network output approximate to the expected output value. No teacher learning includes intensive learning and unsupervised learning. In intensive learning, the learning of output is accomplished by minimizing the continuous effect of the external environment.

\subsection{Learning of BP Neural Network}

BP neural network refers to the learning process that contains the forward propagation of information and the algorithm of error back propagation. Each neuron in the input layer is responsible for receiving the input information from the outside world, and passed to each neuron in the middle layer; the middle layer neurons is the internal information processing layer, responsible for information transformation, can be designed as a single hidden layer or multi hidden layer structure; the last hidden layer to the output layer transfer information. After further treatment after completion of a forward propagation process of learning, from the output layer to the external output information processing. When the actual output does not match the desired output, it enters the back-propagation phase of the error. The error is acted on the input layer by the output layer, and the weights of each layer are corrected respectively in the way of the error gradient descending, and gradually back to the hidden layer and the input layer. After several information spread forward and backward error propagation process, is a process of constantly adjust the weights, which is also a process of neural network training, the process has been carried out to the output errors of the network can be reduced to an acceptable level, or to achieve the predetermined learning times will stop. The neurons in the layer and layer are connected by the full interconnection, and the neurons in the hidden layer are not connected by the corresponding weight coefficients. Therefore, the BP network can be regarded as a highly nonlinear mapping from input to output. In the mapping, topological invariance is maintained. If the number of neurons in the hidden layer is enough, the BP network can simulate any rational function. Because the BP network can establish a nonlinear mapping between multiple continuous inputs and one or more continuous outputs, it is often used for intelligent prediction. Thus, we use the 
BP neural network to simulate the grid resource scheduling process.

\section{Face Recognition Algorithm Based on Computer Neural Network}

\subsection{Face Image Detection}

Face image detection is to judge whether the face is contained in the input image, if so, if it has, it will give the location and size of the face and so on, and separate the face area from the background. Face patterns contain rich pattern features, such as color features, structural features and histogram features. There are many mature algorithms for detection, which are no longer mentioned here. For the sake of simplicity, this article uses face images in the database directly. Because the learning rate is fixed, the convergence rate of the network is slow, and it takes longer training time. This long and uncertain training process, for a relatively simple problem, sometimes requires hundreds or even thousands of times of learning to converge. For complex problems, it may take longer to train the network. The BP algorithm can make the weights converge to a certain value, and cannot guarantee the convergence to the global minimum value and easily fall into the local minimum. During training, when the weighting is large, it may force all or most of the nodes to have larger weights and outputs, and thus work in the saturation region of the excitation function, when the derivative of the excitation function is in a very small area. In the formula of calculating the correction of weights, each layer error is proportional to the derivative of the excitation function. When the derivative tends to zero, the error of each layer tends to zero, which is equivalent to the stagnation of the regulation process. It is easy to cause the network to not reach the specified error, and the training is not available. The number of layers of the hidden layer of the network and the selection of the number of nodes in each layer have not yet been guided theoretically, but are determined according to experience. Therefore, the network often has a lot of redundancy, and the network learning time is added invisibly.

\subsection{Face Image Preprocessing}

Preprocessing is an important link in the process of face recognition. Due to the different environment of image acquisition, such as the degree of darkness of light and the performance of the device, there are many shortcomings such as noise and contrast. In addition, distance and distance, the size and location of the face in the middle of the whole image are uncertain. To ensure the consistency of face size, position and image quality in face image, it is necessary to preprocess the image. Because the original image will be restricted by various conditions and random interference during the process of generation and transmission, it is necessary to pre-grate the image, such as gray correction and noise filtering before face recognition. The purpose of preprocessing is to eliminate the interference of noise and redundant information, to reduce the impact of environmental factors on the image, and to highlight the important information of the image. The general approach is to convert the original image into a gray matrix first. Face image preprocessing mainly includes face centralization, face image enhancement, and normalization and so on. Face straighten is to get the correct face image. Image enhancement is to improve the quality of face images, not only in visual clearer images, but also in computer processing and recognition. The goal of normalization is to achieve a standardized face image with the same size and the same range of gray value.

\subsection{Feature Extraction of Face Image}

When the original image is converted to a grayscale image, the amount of data contained is relatively large. If we directly calculate it, the computation will be very large. Therefore, we need further processing gray image to extract its eigenvalues. Image feature extraction and classification for face feature information extraction, including geometric features commonly used in describing face: eyebrow thickness, the vertical distance between the eyebrows and eyes; elevn parameters describing the curvature of the eyebrows; the width of the nose, the nose of the vertical position; the vertical distance of the upper and lower lips, mouth width the thickness of the mouth, chin; description parameters of shape; the nostrils face wide position; the middle position of the nose and 
eyes wide face. The methods of facial feature extraction can be classified into two categories: one is knowledge representation, and the other is algebraic representation or statistical learning. The geometric characteristics of the extraction of knowledge depends on prior knowledge based, has many limitations and inaccuracy of learning methods based on statistical methods to extract the statistical characteristics of all training images, facial feature extraction does not require each face, will be more effective, so it has become the mainstream of current method. No matter what kind of facial feature extraction method is to divide the image into small areas, from high dimensional space to a representation expressed in low dimensional space, the image processing is selected by adding low dimensional space can best represent the image feature data, which can reduce the dimension of feature.

\subsection{Matching and Recognition of Face Image}

The facial geometry topology a priori knowledge, use structure method based on Extraction of facial features of main organs in the knowledge level, will face with a set of geometric feature vector representation, recognition, feature vector is attributed to the judgment based on Euclidean distance is the most commonly used method. The recognition method based on geometric features has the advantages of small storage and insensitive to light. There are also some problems in this method. It is difficult to extract stable features from images, poor robustness to strong expression changes and posture changes, and cause partial information loss, which is suitable for rough classification. The face recognition methods based on statistical features include the feature face method and the hidden Markov model method. The method of statistical recognition means that the face is represented by the algebraic feature vector. The algebraic feature is determined by the gray distribution of the image itself. It describes the memory information of the image. It is put forward by various algebraic transformation and matrix decomposition of the image grayscale. After the image gray preprocessing and feature extraction, it is necessary to design an algorithm to classify the features of the input and realize the face recognition. Face recognition is to compare the facial features that need to be recognized with the obtained facial feature templates, and to identify the face's identity information according to the similarity degree. We regard human faces as a two-dimensional grayscale change template to capture and describe the features of the face. We mainly use some standard data statistics techniques, and the operation is more complicated.

\section{Conclusions}

Face recognition is a difficult research topic, and it is still in the exploration stage. In this paper, the feature extraction and recognition of face are realized by using the advantages of strong learning ability and strong classification ability of BP neural network. There are some defects in the practical application of BP network. We should improve the BP algorithm to improve the accuracy of recognition.

\section{Acknowledgements}

The paper is supported by Special Plan Project of Science Research of Educational Department of Shaanxi Province (Grant No. 17JK1059).

\section{References}

[1] Xing Ling, Feng Qian, Mu Guowang. Face recognition based on convolution neural network and PCA [J]. Journal of Hebei University of Technology, 2016, 45(5): 44-49.

[2] Chen Yaodan, Wang Lianming. Convolutional neural network for face recognition [J]. Journal of Northeast Normal University (Natural Science Edition), 2016, 48(2): 70-76.

[3] Yu Daling, Huang Hongqiong. A face recognition method based on CNN [J]. Microcomputer \& Its Applications, 2017, 36(14): 49-51+56. 
[4] Lu Guanming, He Jiali, Yan Jingjie, et al. Convolutional neural network for facial expression recognition [J]. Journal of Nanjing University of Posts and Telecommunications (Natural Science Edition), 2016, 36(1): 16-22. 